\title{
Lo que hemos aprendido durante la pandemia: la cloroquina
}

\author{
Víctor M. Camarillo-Nava ${ }^{1 *}$, José V. Rosas-Barrientos ${ }^{2}$, Norma A. Hernández-Pineda ${ }^{3}$, \\ Francisco J. Moreno-Rodríguez ${ }^{4}$, Ricardo E. Aguilar-Vargas ${ }^{5}$, Gustavo D. Quiroz-Cortés ${ }^{6}$, \\ Elizabeth López-Rojas ${ }^{7}$, Jorge L. Zendejas-Villanueva ${ }^{8}$ y José A. Zamudio-González ${ }^{9}$
}

${ }^{1}$ Departamento de Investigación en Salud, Órgano de Operación Administrativa Desconcentrada DF Norte, Instituto Mexicano del Seguro Social; ${ }^{2}$ Departamento de Medicina Interna, Hospital 1.0 de Octubre, Instituto de Seguridad y Servicios Sociales de los Trabajadores del Estado; ${ }^{3}$ Departamento de Educación en Salud, Hospital General de Zona 24, Instituto Mexicano del Seguro Social; ${ }^{4}$ Departamento de Cardiología, Hospital General de Zona 24, Instituto Mexicano del Seguro Social; ${ }^{5}$ Departamento de Medicina Interna, Hospital General de Zona 24, Instituto Mexicano del Seguro Social; ${ }^{\circ}$ Departamento de Investigación en Salud, Órgano de Operación Administrativa Desconcentrada DF Norte, Instituto Mexicano del Seguro Social; ' Departamento de Planeación y Enlace Institucional, Órgano de Operación Administrativa Desconcentrada DF Norte, Instituto Mexicano del Seguro Social; ${ }^{8}$ Departamento de Servicios de Prestaciones Médicas, Órgano de Operación Administrativa Desconcentrada DF Norte, Instituto Mexicano del Seguro Social; ${ }^{\circ}$ Órgano de Operación Administrativa Desconcentrada DF Norte, Instituto Mexicano del Seguro Social. Ciudad de México, México

\section{Resumen}

Introducción: A inicios de la pandemia por el nuevo coronavirus 2 del síndrome respiratorio agudo grave (SARS-CoV-2) se estableció que el tratamiento con cloroquina podía ser efectivo para contener la replicación viral in vitro, pero aún existe controversia sobre su efectividad en humanos para disminuir la mortalidad por enfermedad por coronavirus 2019 (COVID-19) en pacientes graves. Objetivo: Evaluar la mortalidad asociada al uso de cloroquina en pacientes con infección grave por SARS-CoV-2. Métodos: Estudio de cohorte retrospectiva, en un hospital de 2. ${ }^{\circ}$ nivel. Se revisaron 171 expedientes de pacientes hospitalizados por COVID-19 grave, se analizó el riesgo de mortalidad por uso de cloroquina. Se analizaron datos sociodemográficos, patologías previas, días desde el inicio de los síntomas hasta la atención médica, días de estancia hospitalaria, parámetros respiratorios; gasometría, citometría hemática, deshidrogenasa láctica, proteína C reactiva (PCR), tratamiento antiviral, tromboprofilaxis, uso de esteroides y uso de antibióticos. Se utilizó estadística descriptiva, inferencial, modelos univariantes y multivariantes. Resultados: El uso de cloroquina, la edad avanzada, la obesidad y la PCR baja se asociaron con una mayor probabilidad de muerte en el análisis univariante. La edad (hazard ratio [HR]: 0.976; intervalo de confianza del 95\% [IC 95\%]: 0.959-0.993), no padecer hipertensión (HR: 0.788; IC 95\%: 0.620-1.00) y el uso de cloroquina (HR: 2.07; IC 95\%: 1.2-3.4), fueron factores de riesgo significativos asociados con la muerte en los casos con COVID-19 grave. Conclusiones: En este estudio encontramos evidencia para recomendar evitar el uso de cloroquina en pacientes adultos con COVID-19 grave debido al aumento en el riesgo de mortalidad hasta dos veces más en comparación con los que no se les administró.

Palabras clave: Cloroquina. SARS-CoV-2. Mortalidad. COVID-19 severa.

\section{Correspondencia:}

*Víctor M. Camarillo-Nava

E-mail: drcamarillonava@gmail.com

DOI: 10.24875/REIE.21000079

Fecha de recepción: 19-07-2021

Disponible en internet: 10-11-2021

Rev Educ Investig Emer. 2022;4(1):11-20 www.medicinadeemergencias.com

2604-6520 @ 2021 Sociedad Mexicana de Medicina de Emergencias, AC. Publicado por Permanyer México SA de CV. Este es un artículo open access bajo la licencia CC BY-NC-ND (http://creativecommons.org/licenses/by-nc-nd/4.0/).
} 


\title{
What we have learned during the pandemic: chloroquine
}

\begin{abstract}
Introduction: At the beginning of the pandemic caused by the new SARS-CoV-2, it was established that treatment with chloroquine could be effective to contain viral replication in vitro but there is still controversy about its effectiveness in humans to reduce mortality from COVID-19 in serious patients. Objective: To evaluate the mortality associated with the use of chloroquine in patients with severe COVID-19 infection. Methods: Retrospective cohort study in a $2^{\text {nd }}$ level hospital. 171 records of patients hospitalized for severe COVID-19 infection were reviewed, the risk of mortality due to use of chloroquine was analyzed. Sociodemographic data, previous pathologies, days from the onset of symptoms to medical attention, days of hospital stay, respiratory parameters were analyzed; blood gas, hematic cytometry, DHL, C-reactive protein (CRP), antiviral treatment, thromboprophylaxis, use of steroids and use of antibiotics. Descriptive statistics, inferential univariate and multiple models were used. Results: The use of chloroquine, advanced age, obesity and low CRP were associated with a higher probability of death in the univariate analysis. Age (HR.976; 95\% Cl.,959-.993), not having hypertension (HR.788; 95\% Cl.,6201.00) and use of chloroquine (HR 2.07; 95\% Cl, 1.2-3.4), were significant risk factors associated with death in severe COVID-19 cases. Conclusions: In this study we found evidence to recommend avoiding the use of chloroquine in adult patients with severe COVID-19 due to the increased risk of mortality up to two times higher compared to those who were not administered.
\end{abstract}

Keywords: Chloroquine. SARS-CoV-2. Mortality. Severe COVID-19.

\section{Introducción}

A inicios de la pandemia por el coronavirus 2 del síndrome respiratorio agudo grave (SARS-CoV-2) en Wuhan, China, en diciembre de 2019, no se conocía tratamiento específico para su control. Un tratamiento propuesto fue la hidroxicloroquina y la cloroquina (HCQ/CLQ). Se reportó que estos fármacos limitaban la replicación del virus in vitro ${ }^{1}$. Ambos comparten estructuras químicas y mecanismos de acción similares, aunque no se conocían totalmente ante la enfermedad por coronavirus 2019 (COVID-19), ya que parecían ejercer diferentes efectos antivirales ${ }^{2-4}$. Aunque la CLQ se considera clásicamente como un inhibidor de las vías endocíticas por medio de la elevación del pH endosómico ${ }^{5}$, su mecanismo de acción molecular detallado como un compuesto antiviral sigue sin estar claro.

La CLQ podría actuar en varios pasos del ciclo de replicación del coronavirus ${ }^{6}$. Se ha demostrado que los fármacos antipalúdicos inhiben la glucosilación ${ }^{7}$ y fosforilación terminal de la enzima convertidora de angiotensina $2^{6,7}$, que actúa como un receptor de la membrana plasmática tanto para el SARS-CoV ${ }^{8}$, como para el SARS-CoV-2 y elevan el pH en los endosomas, respectivamente.

La HCQ o CLQ con y sin azitromicina se ha asociado con un aumento de la mortalidad (Riesgo Relativo [RR]: 1.27; intervalo de confianza del 95\% [IC 95\%]: 1.041.54; $n=7$ estudios). La HCQ sola no se asoció con una reducción de la mortalidad en los pacientes hospitalizados con COVID-199. Varios estudios encontraron que los pacientes que recibieron $\mathrm{HCQ}$ desarrollaron un intervalo QT corregido (QTc) de 500 milisegundos o más ${ }^{8,10}$. Por otra parte, se asoció también con una mayor necesidad de intensificación de la asistencia respiratoria. No hubo beneficios sobre la mortalidad, la linfopenia o la mejora de la proporción de neutrófilos y linfocitos ${ }^{11}$. Otros estudios evaluaron la eficacia de la CLQ, un ensayo que comparó la terapia se detuvo debido a la preocupación de que la terapia con dosis más altas aumentara la letalidad y prolongación del intervalo QTc. Un análisis intermedio no planificado recomendado por una junta de vigilancia y seguridad de datos independiente de ese estudio encontró que una dosis más alta de difosfato de CLQ durante 10 días se asoció con más efectos tóxicos y letalidad, particularmente afectando la prolongación del intervalo QTc. El tamaño limitado de la muestra no permitió que el estudio mostrara ningún beneficio en general con respecto a la eficacia del tratamiento ${ }^{12}$.

La evidencia sobre los beneficios y daños del uso de HCQ y CLQ para tratar la COVID-19 había sido muy débil y contradictoria hasta ese momento ${ }^{13,14}$.

Aunque el tratamiento con CLQ se indicó en un principio que podía ser efectivo para contener la replicación viral in vitro ${ }^{15}$ y disminuir la mortalidad por la COVID-19 en pacientes graves, posteriormente se demostró su verdadera utilidad ${ }^{16}$. El objetivo de este estudio fue evaluar el impacto de la administración de CLQ sobre la mortalidad intrahospitalaria en pacientes graves por COVID-19. 


\section{Material y métodos}

Estudio de cohorte retrospectivo, analítico. Se analizaron los expedientes de pacientes hospitalizados en área de urgencias de un hospital de $2 .^{\circ}$ nivel, con diagnóstico de COVID-19 mediante prueba de reacción en cadena de la polimerasa transcriptasa reversa procesada en laboratorios con reconocimiento del Instituto de Diagnóstico y Referencia Epidemiológicos (InDRE $)^{17}$. Se incluyeron pacientes mayores de 18 años, de ambos sexos, con criterios de infección grave, con manejo durante su estancia hospitalaria con CLQ y que ingresaron durante el mes de mayo 2020. Se definió enfermedad COVID-19 grave $^{18}$ según resultados de imagenología y marcadores inflamatorios: dímero $\mathrm{D}$ mayor de $1,000 \mathrm{mg} / \mathrm{dl}$, creatina fosfocinasa (CPK) dos veces por arriba del parámetro de referencia, proteína $C$ reactiva $>100 \mathrm{mg} / \mathrm{d}$, deshidrogenasa láctica $(\mathrm{DHL})$ $>245 \mathrm{Ul}$ y cuenta de leucocitos $<800 \mathrm{cel} / \mathrm{mm}^{3}$. Imagenología: áreas extensas de incremento de la opacidad y consolidaciones francas del espacio aéreo, afección bilateral y de predominio en lóbulos inferiores. Se definió linfocitopenia ${ }^{19,20}$ como $<800 / \mathrm{mm}^{3}$.

Se analizaron datos sociodemográficos, patologías previas, días transcurridos desde el inicio de los síntomas hasta la atención médica, días de estancia hospitalaria, parámetros respiratorios, citometría hemática, $\mathrm{DHL}$, proteína $\mathrm{C}$ reactiva (PCR), tratamiento antiviral, tromboprofilaxis, uso de esteroides y antibióticos administrados. No se contaba con trazo electrocardiográfico en todos los casos, motivo por el cual no se incluyó como variable de estudio. El criterio de exclusión fue la contraindicación absoluta de CLQ.

Se calculó el tamaño de muestra con la fórmula de una proporción con un nivel de confianza al 95\%, potencia del $80 \%$, frecuencia observada en la mortalidad de un $10 \%$ de diferencia entre los usuarios y no usuarios de CLQ y un límite de confianza del 5\%. El tamaño de muestra fue de 171 expedientes. Para conocer el tipo de distribución de datos cuantitativos se utilizó la prueba de Kolmogorov-Smirnoff ${ }^{21}$. Se usó la prueba t de Student, $U$ de Mann Whitney, $\chi^{2}$ o la prueba exacta de Fisher para comparar las diferencias entre los supervivientes y los no supervivientes cuando fue apropiado.

Para explorar los factores de riesgo asociados a la muerte intrahospitalaria, se utilizaron modelos univariantes y multivariantes. Se seleccionó el uso o no de $C L Q$, esteroides, enfermedades previas, edad, linfocitos y relación $\mathrm{PaO}_{2} / \mathrm{FIO} 2$ (presión arterial de oxígeno/ fracción inspirada de oxígeno) como variables para el modelo multivariante.

En el caso de la regresión logística se excluyeron del modelo aquellas variables que en el análisis univariante sus diferencias entre los grupos no fueron estadísticamente significativas, si su precisión no estaba confirmada o si el número de eventos era demasiado pequeño para calcular los riesgos relativos. En todos los análisis comparativos se designó el valor de p menor o igual a 0.05 como estadísticamente significativo. Para el procesamiento de los datos se utilizó el programa estadístico SPSS V.24.

El estudio se apegó a la Declaración de Helsinki, principios bioéticos básicos, pautas CIOMS y al Reglamento de la Ley General de Salud en Materia de Investigación para la Salud. Esta investigación se clasificó como riesgo mínimo y se otorgó el registro institucional: R-2020-3509-052.

\section{Resultados}

Fueron hospitalizados 558 pacientes por diagnóstico confirmado de COVID-19 grave en el periodo de estudio. La mortalidad global fue de 43.9\% (245 muertes), se revisaron 320 expedientes y se excluyeron 149 por no contar con información clave. Incluimos 171 expedientes en el análisis final (67 con uso de CLQ [39\%] y 104 sin él [61\%]). La media de edad fue de 58.5 años (rango intercuartílico [RIQ]: 20-95), el 71.9\% fueron hombres (123 sujetos). Las comorbilidades más frecuentes se observan en la tabla 1.

Los síntomas reportados por orden de frecuencia fueron: fiebre, tos, disnea y mialgias; 167 pacientes recibieron antibióticos $(97.7 \%)$ y $2(1.2 \%)$ recibieron antivirales (lopinavir/ritonavir). La comparación de las características, síntomas y el tratamiento de los pacientes con y sin el uso de CLQ se muestran en la tabla 1. Se produjo linfocitopenia en 70 (48.9\%) pacientes de 143 que contaron con estudios de hemograma completo. El recuento inicial de linfocitos fue significativamente mayor en los supervivientes que en los no supervivientes $\left(1,100 / \mathrm{mm}^{3}\right.$ vs. $770 / \mathrm{mm}^{3}$ respectivamente); en el recuento subsecuente se mantuvieron los niveles estables en los supervivientes y se observó linfocitopenia grave en los no supervivientes. Los niveles de PCR y CPK estaban claramente elevados en los pacientes que fallecieron en comparación con los supervivientes a lo largo del curso clínico y aumentaron con el deterioro de la enfermedad. La DHL se mantuvo elevada tanto para los supervivientes como para los fallecidos (Tabla 2). 
Tabla 1. Datos generales de pacientes hospitalizados en el mes de mayo por COVID-19 grave, por grupo de tratamiento en HGZ 24 IMSS

\begin{tabular}{|c|c|c|c|c|c|c|}
\hline & \multirow{2}{*}{$\begin{array}{c}\text { Total } \\
\text { (n= 171) }\end{array}$} & \multicolumn{2}{|c|}{ Tratados con cloroquina (67) } & \multicolumn{2}{|c|}{ Tratados sin cloroquina (104) } & \multirow[t]{2}{*}{$\mathbf{p}^{*}$} \\
\hline & & $\bar{x}(D E)$ & Rango & $\bar{x}(\mathrm{DE})$ & Rango & \\
\hline Edad & $58( \pm 13.7)$ & $58.5( \pm 13.3)$ & $20-95$ & $58.4( \pm 14)$ & $29-84$ & $0.988^{*}$ \\
\hline IMC & $29.9( \pm 6.1)$ & $30( \pm 4.7)$ & $17.7-69.8$ & $29.8( \pm 7)$ & $21.2-40.5$ & $0.871^{*}$ \\
\hline & n (\%) & n & $\%$ & n & $\%$ & $\mathbf{p}^{\dagger}$ \\
\hline $\begin{array}{l}\text { Sexo } \\
\text { Hombre } \\
\text { Mujer }\end{array}$ & $\begin{array}{l}123(71.9) \\
48(28.1)\end{array}$ & $\begin{array}{l}51 \\
16\end{array}$ & $\begin{array}{l}76 \% \\
24 \%\end{array}$ & $\begin{array}{l}72 \\
32\end{array}$ & $\begin{array}{l}69 \% \\
31 \%\end{array}$ & 0.328 \\
\hline $\begin{array}{l}\text { Patologías más frecuentes } \\
\text { HAS } \\
\text { DM } \\
\text { EPOC/asma } \\
\text { Cardiopatía } \\
\text { IRC } \\
\text { Cáncer } \\
\text { Artritis reumatoide } \\
\text { Insuficiencia venosa } \\
\text { Otras } \\
\text { S/P }\end{array}$ & $\begin{array}{c}64(37.4) \\
58(33.9) \\
9(4.8) \\
6(3.6) \\
4(2.4) \\
3(1.8) \\
3(1.8) \\
2(1.2) \\
7(4.2) \\
52(31.2)\end{array}$ & $\begin{array}{c}24 \\
21 \\
4 \\
1 \\
1 \\
2 \\
1 \\
0 \\
3 \\
21\end{array}$ & $\begin{array}{c}36 \% \\
32 \% \\
5.9 \% \\
1.5 \% \\
1.5 \% \\
2.9 \% \\
1.5 \% \\
0 \\
4.4 \% \\
31.2 \%\end{array}$ & $\begin{array}{c}40 \\
37 \\
5 \\
5 \\
3 \\
1 \\
2 \\
2 \\
4 \\
31\end{array}$ & $\begin{array}{c}38.5 \% \\
36 \% \\
4.8 \% \\
4.8 \% \\
2.7 \% \\
0.9 \% \\
1.9 \% \\
1.9 \% \\
3.8 \% \\
29.8 \%\end{array}$ & $\begin{array}{c}0.728 \\
0.568 \\
0.73 \\
0.25 \\
0.55 \\
0.32 \\
0.83 \\
0.36 \\
0.56 \\
0.83\end{array}$ \\
\hline $\begin{array}{l}\text { Tratamiento establecido } \\
\text { Esteroide (metilprednisolona) } \\
\text { Antibióticos } \\
\text { Antiviral Lopinavir/ritonavir } \\
\text { Tromboprofilaxis } \\
\text { (HBPM) }\end{array}$ & $\begin{array}{c}73(42 \%) \\
167(97 \%) \\
2(1.16 \%) \\
158(92 \%)\end{array}$ & $\begin{array}{c}24 \\
66 \\
1 \\
64\end{array}$ & $\begin{array}{c}35.8 \% \\
98.5 \% \\
1.4 \% \\
95.5 \%\end{array}$ & $\begin{array}{c}49 \\
101 \\
1 \\
94\end{array}$ & $\begin{array}{c}47 \% \\
97.1 \% \\
0.96 \% \\
90.3 \%\end{array}$ & $\begin{array}{c}0.145 \\
0.554 \\
0.632^{*} \\
0.253^{*}\end{array}$ \\
\hline
\end{tabular}

El tiempo medio desde el ingreso hospitalario hasta el alta fue de 8.5 días (RIQ: 2-31), mientras que el tiempo medio hasta la muerte fue de 6 (RIQ: 1-50) (Tabla 3). Treinta y seis pacientes $(21 \%)$ requirieron ventilación mecánica invasiva, de los cuales fallecieron $36(100 \%)$. La mediana de tiempo desde el inicio de la enfermedad hasta la ventilación mecánica invasiva fue de 12 días (10-21) y la mediana de días en ventilación fue de 3 (RIQ: 1-21). La sepsis fue la complicación más frecuente en 16 pacientes (9.3\%), seguida de insuficiencia renal en $15(8.8 \%)$ y complicaciones neurológicas en 6 (3.5\%). La frecuencia de complicaciones fue mayor en los no supervivientes 31 (18\%) que en los supervivientes 6 (3.5\%). El tiempo promedio para acudir a atención médica, inicio de la ventilación, duración, muerte $o$ alta (Tabla 3).

El tiempo de inicio y la duración del uso sistemático de corticosteroides (metilprednisolona) fue similar entre los grupos, mediana de 3 días en el grupo de sobrevivientes contra mediana de 2 en el grupo de no sobrevivientes $(p=0.665)$ (Tabla 3$)$.
Ochenta y un pacientes fallecieron durante la hospitalización y 90 fueron dados de alta por mejoría. De los 67 pacientes a los que se les administró CLQ murieron 44 (65\%), 78 paciente por cada 1,000 que recibieron dicho tratamiento, en comparación con 37 de los 104 no usuarios de CLQ (35.5\%), 66 por cada 1,000 que no recibieron el tratamiento (odds ratio [OR]: 3.4 ; IC 95\%: 1.8 - 6.6, $p=0.0001$ ).

En el análisis univariante, las probabilidades de muerte intrahospitalaria con el uso de CLQ fueron mayores en comparación con los pacientes a los que no se les suministró. La edad, índice de masa corporal (IMC), leucocitosis, linfocitopenia, CPK, $\mathrm{PaO} 2 / \mathrm{FiO} 2$ y PCR también se asociaron con la muerte. El uso de esteroide y la tromboprofilaxis fueron factores protectores (Tabla 4).

En el modelo de regresión logística, encontramos que el uso de CLQ, la edad avanzada, la obesidad, el índice PaO2/FiO2 y la PCR baja se asociaron con una mayor probabilidad de muerte (Tabla 4).

El análisis de riesgos proporcionales de Cox reveló que la edad (hazard ratio [HR] ajustado: 0.976; IC 95\%: 
Tabla 2. Valores clínicos, bioquímicos y gasométricos al ingreso y a las 72 horas, estudios de imagen, por grupos de tratamiento con y sin cloroquina, y por desenlace final

\begin{tabular}{|c|c|c|c|c|c|c|}
\hline & Total & \multicolumn{2}{|c|}{ Cloroquina } & \multicolumn{2}{|c|}{ Sin cloroquina } & \multirow[t]{2}{*}{$\mathbf{p}^{*}$} \\
\hline & $\bar{x}(D E)$ & $\bar{x}$ & DE & $\overline{\mathrm{x}}$ & DE & \\
\hline FC & $99(20.2)$ & 100.09 & 18.0 & 99.15 & 21.7 & 0.722 \\
\hline FR & $24(6)$ & 24.1 & 5.0 & 24.2 & 6.5 & 0.866 \\
\hline TAM & $88(12.8)$ & 90.6 & 13.7 & 86.5 & 12.3 & 0.053 \\
\hline Temperatura & $37.09(.8)$ & 37.18 & 0.82 & 37.0 & 0.86 & 0.328 \\
\hline \multicolumn{7}{|c|}{ Pa02/FI02 inicial } \\
\hline & & $\bar{x}$ & DE & $\overline{\mathrm{x}}$ & DE & $\mathbf{p}^{*}$ \\
\hline $\mathrm{PaO2/FIO2}$ & $253.2(116)$ & 214 & 117 & 285 & 107 & 0.008 \\
\hline \multicolumn{7}{|c|}{$\mathrm{Pa02/FI02} \mathrm{a} \mathrm{las} 72$ horas } \\
\hline & & $\bar{x}$ & DE & $\bar{x}$ & DE & $\mathbf{p}^{*}$ \\
\hline $\mathrm{Pa02/FIO2}$ & $266(120)$ & 260 & 120 & 293.4 & 104.1 & 0.278 \\
\hline \multicolumn{7}{|c|}{ Biometría hemática inicial } \\
\hline & $n=61$ & mediana & Rango & $n=84$ & Rango & $\mathbf{P}$ \\
\hline Leucocitos & $9.6(4.2)$ & 9.2 & $3-22$ & 8.5 & $3-28$ & $0.735^{\dagger}$ \\
\hline Linfocitos & $1.1(.6)$ & 0.81 & $0.18-2.24$ & 1.13 & $0.11-4.4$ & $0.000^{\dagger}$ \\
\hline Plaquetas & $265(114)$ & 227 & $60-765$ & 263 & $50-628$ & $0.094^{\dagger}$ \\
\hline DHL & $847(172-2,050)$ & 875 & $418-2,050$ & 731 & $172-1,979$ & $0.002^{\dagger}$ \\
\hline CPK & $92(4-2,213)$ & 102 & $28-2,213$ & 85 & $4-1,977$ & $0.055^{\dagger}$ \\
\hline CPK MB & $20(10-1,461)$ & 21 & $10-1,461$ & 18.9 & $10-75$ & $0.104^{\dagger}$ \\
\hline PCR & $84(1.6-520)$ & 3.38 & $2.5-520$ & 3.3 & $1.62-505$ & $0.217^{\dagger}$ \\
\hline \multicolumn{7}{|c|}{ Biometría hemática a las 72 horas } \\
\hline Leucocitos & $9.8(5.4)$ & 11.1 & 6.7 & 8.6 & 3.7 & $0.052^{\dagger}$ \\
\hline Linfocitos & $1.2(.8)$ & 1.0 & 0.6 & 1.3 & 1.0 & $0.007^{\dagger}$ \\
\hline Plaquetas & $347(143)$ & 361 & 134 & 331 & 152 & $0.083^{\dagger}$ \\
\hline DHL & $642(263-2,248)$ & 780 & $379-2,248$ & 589 & $263-1,487$ & $0.001^{\dagger}$ \\
\hline CPK & $70(21-2,578)$ & 95 & $23-2,578$ & 62 & 21-1004 & $0.035^{\dagger}$ \\
\hline CPK MB & $18(8-77)$ & 23 & $12-77$ & 16.6 & $8-50$ & $0.004^{\dagger}$ \\
\hline PCR & $61.4(0.34-36)$ & 102 & $5.3-406$ & 25.3 & $0.34-436$ & $0.002^{\dagger}$ \\
\hline
\end{tabular}

*t de Student.

tU de Mann-Whitney.

n: frecuencia; $\bar{x}$ : media; DE: desviación estándar; $\mathrm{FC}$ : frecuencia cardiaca; $\mathrm{FR}$ : frecuencia respiratoria; $\mathrm{TAM}$ : tensión arterial media; $\mathrm{PaO}_{2}$ : presión arterial de oxígeno; $\mathrm{FIO}$ : fracción inspirada de oxígeno; CPK: creatina fosfocinasa; CPK MB: creatina fosfocinasa fracción mb; DHL: deshidrogenasa láctica; PCR: proteína C reactiva; S/R: sin resultado.

0.959-0.993), no padecer hipertensión (HR ajustado: 0.788; IC 95\%: 0.620-1.00) y el uso de CLQ (HR ajustado: 2.07; IC 95\%: 1.2-3.4) durante la hospitalización fueron factores de riesgo significativos asociados con la muerte en los casos con COVID-19 grave (Tabla 5, Figs. 1 y 2).

\section{Discusión}

Identificamos el tratamiento con CLQ, la edad mayor de 60 años y el padecer hipertensión arterial como factores de riesgo de muerte en adultos hospitalizados 
Rev Educ Investig Emer. 2022;4(1)

Tabla 3. Tiempos medios en la atención de pacientes con COVID-19 grave

\begin{tabular}{|c|c|c|c|c|c|}
\hline & Cloroquina & $\mathbf{N}$ & $\bar{x}$ & DE & $\mathbf{P}$ \\
\hline \multirow[t]{2}{*}{ Días de ventilación } & Uso de cloroquina & 12 & 6 & 4.4 & \multirow[t]{2}{*}{0.038} \\
\hline & No uso cloroquina & 15 & 2.5 & 3.8 & \\
\hline \multirow[t]{2}{*}{ Días de pronación } & Uso de cloroquina & 17 & 4.5 & 2.9 & \multirow[t]{2}{*}{0.028} \\
\hline & No uso cloroquina & 14 & 2.5 & 1.5 & \\
\hline \multirow[t]{2}{*}{ Días de tratamiento con esteroide } & Uso de cloroquina & 16 & 3 & 2.1 & \multirow[t]{2}{*}{0.197} \\
\hline & No uso cloroquina & 11 & 2 & 1.2 & \\
\hline \multirow[t]{2}{*}{ Días de antibiótico } & Uso de cloroquina & 10 & 4.1 & 3.6 & \multirow[t]{2}{*}{0.619} \\
\hline & No uso cloroquina & 9 & 3.3 & 2.8 & \\
\hline \multirow[t]{2}{*}{ Días de tromboprofilaxis } & Uso de cloroquina & 24 & 6.6 & 4.4 & \multirow[t]{2}{*}{0.307} \\
\hline & No uso cloroquina & 26 & 5.0 & 6.1 & \\
\hline \multirow[t]{2}{*}{ Días de estancia intrahospitalaria } & Uso de cloroquina & 25 & 7.0 & 4.9 & \multirow[t]{2}{*}{0.265} \\
\hline & No uso cloroquina & 32 & 4.8 & 8.9 & \\
\hline
\end{tabular}

Tabla 4. Análisis univariante y multivariante en pacientes con COVID-19 grave para la presentación de muerte hospitalaria

\begin{tabular}{|c|c|c|c|c|c|c|}
\hline & \multicolumn{2}{|c|}{ Univariante } & \multirow[t]{2}{*}{$\mathbf{p}$} & \multicolumn{2}{|c|}{ Multivariante } & \multirow[t]{2}{*}{$\mathbf{p}$} \\
\hline & OR & IC $95 \%$ & & HR & IC $95 \%$ & \\
\hline Edad & 2.18 & $1.18-4.02$ & 0.011 & 0.976 & $0.959-0.993$ & 0.006 \\
\hline $\begin{array}{l}\text { Sexo masculino } \\
\text { (vs. femenino) }\end{array}$ & 1.08 & $0.55-2.12$ & 0.801 & 0.976 & $0.76-1.2$ & 0.848 \\
\hline Tabaquismo & 0.91 & $0.46-1.79$ & 0.785 & -- & -- & -- \\
\hline DM & 1.30 & $0.69-2.45$ & 0.413 & -- & -- & -- \\
\hline HAS & 1.30 & $0.70-2.43$ & 0.395 & -- & -- & -- \\
\hline IMC & 2.27 & $1.08-4.75$ & 0.028 & 3.2 & $0.969-10.68$ & 0.056 \\
\hline TAM & 1.13 & $0.15-8.21$ & 0.904 & -- & -- & -- \\
\hline Leucocitosis & 3.57 & $1.72-7.40$ & $<0.0001$ & -- & -- & -- \\
\hline Linfocitopenia & 3.62 & $1.78-7.38$ & $<0.0001$ & 2.16 & $0.64-7.23$ & 0.209 \\
\hline Anemia & 0.67 & $0.22-2.05$ & 0.484 & -- & -- & -- \\
\hline $\mathrm{DHL}$ & 1.70 & $1.47-1.95$ & 0.304 & -- & -- & -- \\
\hline CPK & 2.58 & $1.14-5.82$ & 0.020 & -- & -- & -- \\
\hline $\mathrm{PaO}_{2} / \mathrm{FiO}_{2}$ & 12.4 & $3.89-39.7$ & $<0.0001$ & 14.4 & $2.23-93.0$ & 0.005 \\
\hline PCR > 200 & 0.05 & $0.006-0.44$ & 0.001 & 0.012 & $0.001-0.311$ & 0.008 \\
\hline Esteroide & 0.53 & $0.29-0.99$ & 0.047 & 0.954 & $0.270-3.37$ & 0.942 \\
\hline Cloroquina & 3.46 & $1.81-6.60$ & $<0.0001$ & 2.07 & $1.26-3.40$ & 0.004 \\
\hline Tromboprofilaxis & 0.14 & $0.03-0.67$ & 0.005 & -- & -- & -- \\
\hline
\end{tabular}

COVID-19: enfermedad por coronavirus 2019; OR: odds ratio; IC 95\%: intervalo de confianza del 95\%; HR: hazard ratio; $\overline{\mathrm{X}}$ : media; DE: desviación estándar; TAM: tensión

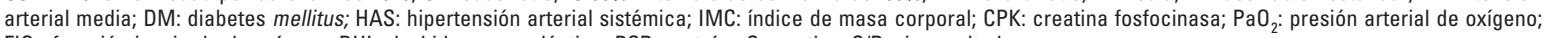
$\mathrm{FIO}_{2}$ : fracción inspirada de oxígeno; DHL: deshidrogenasa láctica; PCR: proteína $\mathrm{C}$ reactiva; S/R: sin resultado. 
Tabla 5. Modelo de Riesgos Proporcionales de Cox para taza de riesgo por mortalidad asociada a cloroquina en paciente con COVID-19 grave

\begin{tabular}{|c|c|c|c|c|c|c|c|c|}
\hline & \multirow[t]{2}{*}{ B } & \multirow[t]{2}{*}{ SE } & \multirow[t]{2}{*}{ WALD } & \multirow[t]{2}{*}{ gl } & \multirow[t]{2}{*}{ Sig } & \multirow[t]{2}{*}{$\operatorname{Exp}(B)$} & \multicolumn{2}{|c|}{$95.0 \%$ CI para $\operatorname{Exp}(B)$} \\
\hline & & & & & & & Inferior & Superior \\
\hline Cloroquina & 0.730 & 0.252 & 8.407 & 1 & 0.004 & 2.076 & 1.267 & 3.401 \\
\hline Edad & -0.024 & 0.009 & 7.428 & 1 & 0.006 & 0.976 & 0.959 & 0.993 \\
\hline Genero & -0.024 & 0.124 & 0.037 & 1 & 0.848 & 0.976 & 0.765 & 1.246 \\
\hline Esteroidessmetilpred & 0.013 & 0.116 & 0.013 & 1 & 0.909 & 1.013 & 0.807 & 1.272 \\
\hline Diabetes & -0.010 & 0.124 & 0.006 & 1 & .937 & 0.990 & 0.777 & 1.262 \\
\hline HAS & -0.239 & 0.122 & 3.834 & 1 & 0.050 & 0.788 & 0.620 & 1.000 \\
\hline
\end{tabular}

COVID-19: enfermedad por coronavirus 2019; SE: error estándar; gl: grados de libertad; p: grado de significación; IC 95\%: intervalo de confianza del 95\%; HAS: hipertensión arterial sistémica.

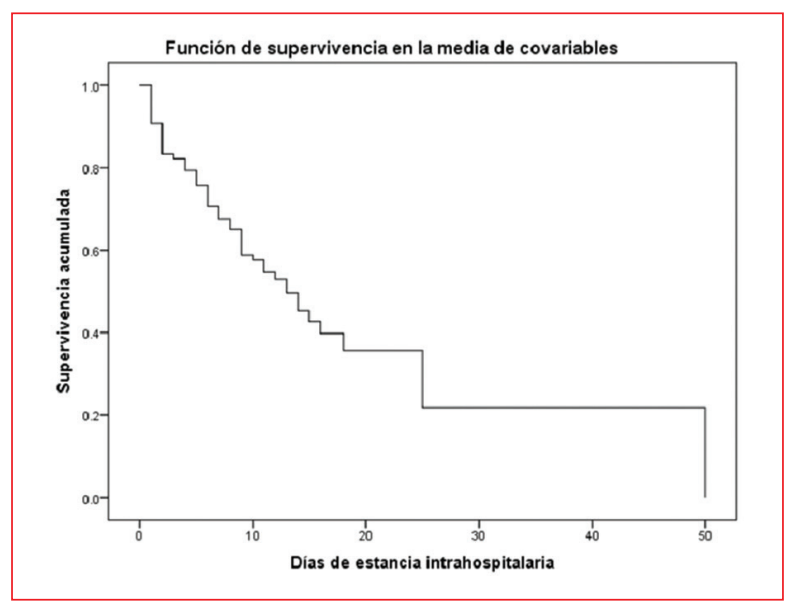

Figura 1. Gráfica de predicción para mortalidad global ajustada por uso de cloroquina, edad mayor de 60 años, uso de esteroides, diabetes mellitus e hipertensión arterial.

por COVID-19 grave. Además, $\mathrm{PaO} 2 / \mathrm{FiO} 2 \leq 200$, linfocitos $\leq 800$, PCR y valores de CPK altos se observaron con mayor frecuencia en los pacientes hospitalizados con COVID-19 grave. La CLQ aumentó el riesgo de muerte hasta dos veces comparado con los que no se les administró.

La edad mayor de 65 años se ha reportado como un factor de riesgo importante para mortalidad por infección por el SARS-CoV-222. Nuestro estudio observó que una edad mayor de 60 años es un factor que se asoció con la muerte. Arshad, et al., encontraron un HR en el modelo de regresión de Cox de 2.6 (IC 95\%: 1.9-3.3), similar al nuestro, el cual fue de 2.18 (IC 95\%: 1.18-4.02). La diabetes mellitus y la

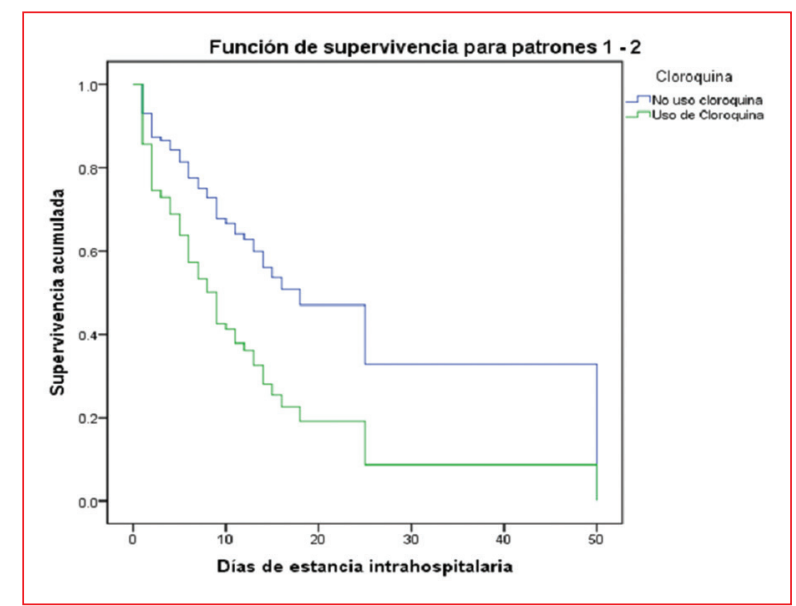

Figura 2. Gráfica de predicción para mortalidad por uso de cloroquina y sin uso, ajustada por uso de cloroquina, edad mayor de 60 años, uso de esteroides, diabetes mellitus e hipertensión arterial.

hipertensión arterial también se han reportado como factores predictores de gravedad y muerte ${ }^{23,24}$, sin embargo en nuestro estudio encontramos que la ausencia de hipertensión es factor protector para mortalidad.

En cuanto al uso de CLQ, actualmente hay siete ensayos clínicos completados y 29 registrados que se centran en HCQ o CLQ como vía terapéutica para COVID-19. De estos, cinco de siete ensayos han mostrado resultados favorables para los pacientes que usan CLQ o HCQ y dos de siete no han mostrado cambios en comparación con el control ${ }^{12}$. Los siete ensayos tenían diversos grados de sesgo y un diseño de estudio deficiente. 
En un ensayo clínico de fase $\mathrm{Ilb}^{12}$, a doble ciego, se evaluó la seguridad y eficacia de dos dosis de CLQ en 81 pacientes hospitalizados con COVID-19 grave. Los pacientes fueron asignados para recibir CLQ en dosis altas (600 mg CLQ dos veces al día durante 10 días) - CLQ en dosis bajas (50 mg dos veces al día el día 1 y una vez al día durante 4 días) (41 [50.6\%] fueron asignados al grupo de dosis alta y 40 [49.4\%] al grupo de dosis baja). El resultado primario fue la reducción de la letalidad en al menos un $50 \%$ en el grupo de dosis alta en comparación con el grupo de dosis baja. Los pacientes incluidos tenían una edad media de 51.1 años, con desviación estándar (DE) de 13,9 años y la mayoría (60 [75.3\%]) eran hombres. En el grupo de dosis alta presentaban mayor edad (edad media: 54.7 \pm 13.7 años frente a $47.4 \pm 13.3$ años) y más enfermedades cardiacas (5 de 28 [17.9\%] frente a 0). La letalidad hasta el día 13 fue del 39.0\% en el grupo de dosis alta (16 de 41$)$ y del $15.0 \%$ en el grupo de dosis baja (6 de 40). El grupo de dosis alta presentó más casos de intervalo QTc superior a 500 milisegundos (7 de 37 [18.9\%]) en comparación con el grupo de dosis baja (4 de 36 [11.1\%]). Los autores sugieren que la dosis más alta de CLQ no debe recomendarse para pacientes críticamente enfermos con COVID-19 debido a sus posibles peligros para la seguridad, especialmente cuando se toman simultáneamente con azitromicina y oseltamivir. Estos hallazgos no se pueden extrapolar a pacientes con COVID-19 no grave. En nuestros resultados, aunque difiere del diseño de estudio encontramos resultados similares, al incrementar el riesgo de mortalidad con el uso de CLQ que los no tratados con el fármaco. Por lo anterior encontramos evidencia para no recomendar el uso de CLQ o HCQ en pacientes adultos con infección por SARS-CoV-2 grave, ya que su uso aumenta el riesgo de mortalidad hasta dos veces cuando se asocia con una edad mayor de 60 años y el antecedente de hipertensión arterial.

En el estudio de 1,376 pacientes de Geleris, et al. ${ }^{26}$, 346 pacientes (25.1\%) presentaron el desenlace final; 180 pacientes fueron intubados, de los cuales 66 murieron posteriormente y 166 murieron sin intubación. En el análisis principal, no hubo asociación significativa entre el uso de HCQ y la intubación o muerte (HR: 1.04; IC 95\%: 0.82-1.32). Dichos resultados contrastan con los nuestros, aunque el tratamiento primario en ese estudio fue la $\mathrm{HCQ}$, nuestro estudio sí demostró evidencia de riesgo en la mortalidad con el uso del antipalúdico. Incluso la revista The Lancet emitió un artículo de retractación sobre el beneficio de CLQ e HCQ en el que concluyen que no fue posible confirmar un beneficio cuando se usan solos o en combinación con un macrólido. Cada uno de los regímenes farmacológicos usados en el estudio se asoció con una disminución de la supervivencia hospitalaria y una mayor frecuencia de arritmias ventriculares ${ }^{27}$. Por su parte, en el estudio SOLIDARITY ${ }^{25}$, conducido por la Organización Mundial de la Salud, se produjo la muerte en 104 de 947 pacientes que recibieron HCQ y en 84 de 906 que recibieron su control (tratamiento estándar sin HCQ) (razón de tasas: 1.19; IC 95\%: 0.89-1.59; $p=0.23$ ), los autores concluyen que estos regímenes de HCQ tuvieron poco o ningún efecto en los pacientes hospitalizados con COVID-19, como lo indica la mortalidad general. A diferencia de nuestro estudio, sí se encontró un aumento en la mortalidad intrahospitalaria con el uso de CLQ respecto a sin su uso, sin embargo la diferencia estriba en el diseño del estudio, en el cual en nuestro estudio no pudimos controlar otras variables como la misma aplicación de la CLQ.

Por otra parte, el estudio RECOVERY ${ }^{28}$, financiado por los Institutos de Salud del Reino Unido, reportó que la muerte a 28 días ocurrió en 421 pacientes (27.0\%) en el grupo de HCQ y en 790 (25.0\%) en el grupo de atención habitual (cociente de tasas: 1.09; IC 95\%: 0.97-1.23; $p=0.15$ ). Se observaron resultados consistentes en todos los subgrupos de pacientes estudiados. Los resultados sugieren que los pacientes del grupo de $\mathrm{HCQ}$ tenían menos probabilidades de ser dados de alta vivos del hospital a los 28 días que los del grupo de atención habitual (59.6 vs. $62.9 \%$; razón de tasas: 0.90; IC 95\%: 0.83-0.98). Finalmente concluyen que entre los pacientes hospitalizados con $\mathrm{CO}$ VID-19, los que recibieron HCQ no tuvieron menor incidencia de muerte a los 28 días que los que recibieron la atención habitual. Dichos resultados son diferentes a nuestros hallazgos y quizá se debe a un sesgo de ejecución al administrar el fármaco a los pacientes más graves, lo cual fue una variable que salió del control de los investigadores al ser un estudio retrospectivo.

Aunque los estudios observacionales como este no pueden explicar completamente los factores de confusión, nuestros hallazgos sugieren no solo una ausencia de beneficio terapéutico, sino también un daño potencial. En nuestro análisis se evidenció el aumento de riesgo de muerte en los pacientes a los cuales se les administró CLQ en comparación con aquellos a los que no se les administró. En el análisis de regresión logística el riesgo se eleva hasta 13 veces más con IC significativo. El HR en el análisis de regresión de riesgos proporcionales de Cox el riesgo fue de dos veces más, ajustado por edad y presencia de hipertensión. 
Una de las debilidades de nuestro estudio fue el diseño retrospectivo, lo que lo hacía lábil a sesgos de diversa naturaleza. El observar un aumento importante en la mortalidad en los usuarios de CLQ puede tratarse de un sesgo de selección y es posible que la naturaleza del riesgo no sea tan elevada. Es muy probable que los clínicos hubieran decidido dar CLQ a los pacientes más graves. Por lo anterior sugerimos un sesgo en la selección del tratamiento, por lo que la mortalidad estaría determinada por la naturaleza de las cohortes y no solo por el uso de la CLQ.

\section{Conclusiones}

Nuestros hallazgos sugieren no solo una ausencia de beneficio terapéutico, sino que encontramos evidencia para formular la recomendación de evitar el uso de CLQ en pacientes adultos con COVID-19 grave, ya que el análisis univariante y multivariante evidenció el aumento en el riesgo de mortalidad hasta dos veces más que en pacientes sin su uso y con condiciones patológicas preexistentes.

\section{Agradecimientos}

Agradecemos a los médicos pasantes en servicio social por concluir la recolección de los datos: Luis Alan Mendoza Godínez, Leticia Yatzil Rivera Medina y Luis Gerardo Pérez Díaz.

\section{Financiamiento}

Los autores no recibieron patrocinio para llevar a cabo este artículo.

\section{Conflicto de intereses}

Los autores declaran que no tienen intereses económicos en competencia 0 relaciones personales que puedan haber influido en el trabajo informado en este documento.

\section{Responsabilidades éticas}

Protección de personas y animales. Los autores declaran que los procedimientos seguidos se conformaron a las normas éticas del comité de experimentación humana responsable y de acuerdo con la Asociación Médica Mundial y la Declaración de Helsinki.
Confidencialidad de los datos. Los autores declaran que han seguido los protocolos de su centro de trabajo sobre la publicación de datos de pacientes.

Derecho a la privacidad y consentimiento informado. Los autores declaran que en este artículo no aparecen datos de pacientes.

\section{Bibliografía}

1. Meo SA, Klonoff DC, Akram J. Efficacy of chloroquine and hydroxychloroquine in the treatment of COVID-19. Eur Rev Med Pharmacol Sci. 2020;24(8):4539-47.

2. Shittu MO, Afolami OI. Improving the efficacy of chloroquine and hydroxychloroquine against SARS-CoV-2 may require zinc additives - A better synergy for future COVID-19 clinical trials. Infez Med. 2020;28(2):192-7.

3. Savarino L, Di Trani I, Donatelli R, Cauda A. Cassone. New insights into the antiviral effects of chloroquine. Lancet Infect Dis. 2006;6(2):67-9.

4. Sinha N, Balayla G. Hydroxychloroquine and COVID-19. Postgrad Med J. 2020;96(1139):550-5.

5. Mauthe M, Orhon I, Rocchi C, Zhou X, Luhr M, Hijlkema KJ, et al. Chloroquine inhibits autophagic flux by decreasing autophagosome-lysosome fusion. Autophagy. 2018;14(8):1435-55.

6. Li W, Moore MJ, Vasilieva N, Sui J, Wong SK, Berne MA, et al. Angiotensin-converting enzyme 2 is a functional receptor for the SARS coronavirus. Nature. 2003;426(6965):450-4.

7. Zheng Z, Peng F, Xu B, Zhao J, Liu H, Peng J, et al. Risk factors of critical \& mortal COVID-19 cases: A systematic literature review and meta-analysis. J Infect. 2020;81(2):e16-e25

8. Chen J, Liu D, Li L, Liu P, Xu Q, Xia L, et al. [A pilot study of hydroxychloroquine in treatment of patients with moderate COVID-19. Zhejiang Da Xue Xue Bao Yi Xue Ban. 2020;49(2):215-9.

9. Fiolet T, Guihur A, Rebeaud ME, Mulot M, Peiffer-Smadja N, Mahamat-Saleh Y. Effect of hydroxychloroquine with or without azithromycin on the mortality of coronavirus disease 2019 (COVID-19) patients: a systematic review and meta-analysis. Clin Microbiol Infect. 2021;27(1):19-27.

10. Bessière $F$, Roccia $H$, Delinière $A$, Charrière $R$, Chevalier $P$, Argaud $L$, et al. Assessment of QT Intervals in a case series of patients with coronavirus disease 2019 (COVID-19) infection treated with hydroxychloroquine alone or in combination with azithromycin in an intensive care unit. JAMA Cardiol. 2020;5(9):1067-9.

11. Barbosa J, Kaitis D, Freedman R, Le K, Lin X. Clinical outcomes of hydroxychloroquine in hospitalized patients with COVID-19: a quasi-randomized comparative study [Internet]. N Engl J Med; 2020. Disponible en: https://www.sefq.es/_pdfs/NEJM_Hydroxychlorquine.pdf

12. Silva Borba MG, Almeida Val FF, Souza Sampaio V, Araujo Alexandre MA, Cardoso Melo G, Brito M, et al. Effect of high vs. low doses of chloroquine diphosphate as adjunctive therapy for patients hospitalized with severe acute respiratory syndrome coronavirus 2 (SARS-CoV-2) infection: A randomized clinical trial. JAMA Netw Open. 2020;3(4):e208857.

13. Hernandez AV, Roman YM, Pasupuleti V, Barboza JJ, White CM. Hydroxychloroquine or chloroquine for treatment or prophylaxis of COVID-19: A living systematic review. Ann Intern Med. 2020;173:287-96.

14. Walls AC, Park YJ, Tortorici MA, Wall A, McGuire AT, Veesler D. Structure, function, and antigenicity of the SARS-CoV-2 spike glycoprotein. Cell. 2020;181(2):281-92.

15. Quiros Roldan E, Biasiotto G, Magro P, Zanella I. The possible mechanisms of action of 4-aminoquinolines (chloroquine/hydroxychloroquine) against SARS-CoV-2 infection (COVID-19): A role for iron homeostasis? Pharmacol Res. 2020;158:104904.

16. Elavarasi A, Prasad M, Seth T, Sahoo RK, Madan K, Nischal N, et al. Chloroquine and hydroxychloroquine for the treatment of COVID-19: a systematic review and meta-analysis. J Gen Intern Med. 2020;35(11):330814.

17. Gobierno de México. Instituto de Diagnóstico y Referencia epidemiológicos Dr. Manuel Martínez Báez [Internet] Gobierno de México, Instituto de Diagnóstico y Referencia epidemiológicos Dr. Manuel Martínez Báez. Disponible en: https://www.gob.mx/salud/acciones-y-programas/instituto-de-diagnostico-y-referencia-epidemiologicos-indre

18. Cao B, Wang Y, Wen D, Liu W, Wang J, Fan G. A trial of lopinavir-ritonavir in adults hospitalized with severe Covid-19. N Engl J Med. 2020;382(19):1787-99.

19. Chiappini E, Galli L, Azzi A, Resti M, Bonsignori F, de Martino M. Lymphocytopenia as a marker for pandemic influenza A/H1N1 2009 virus infection in children. J Med Virol. 2011;83(1):1-4

20. Aslam M. Introducing Kolmogorov-Smirnov tests under uncertainty: An application to radioactive data. ACS Omega. 2019;5(1):914-7. 
Rev Educ Investig Emer. 2022;4(1)

21. Arshad S, Kilgore P, Chaudhry ZS, Jacobsen G, Wang DD, Huitsing K et al. Treatment with hydroxychloroquine, azithromycin, and combination in patients hospitalized with COVID-19. Int J Infect Dis. 2020;97:396-403.

22. Huang I, Lim MA, Pranata R. Diabetes mellitus is associated with increased mortality and severity of disease in COVID-19 pneumonia - A systematic review, meta-analysis, and meta-regression. Diabetes Metab Syndr. 2020;14(4):395-403

23. Zaki N, Alashwal H, Ibrahim S. Association of hypertension, diabetes, stroke, cancer, kidney disease, and high-cholesterol with COVID-19 disease severity and fatality: A systematic review. Diabetes Metab Syndr. 2020;14(5):1133-42.

24. Chowdhury MS, Rathod J, Gernsheimer J. A rapid systematic review of clinical trials utilizing chloroquine and hydroxychloroquine as a treatment for COVID-19. Acad Emerg Med. 2020;27(6):493-504.
25. Geleris J, Sun Y, Platt J, Zucker J, Baldwin M, Hripcsak G, et al. Observational study of hydroxychloroquine in hospitalized patients with $\mathrm{Co}$ vid-19. N Engl J Med. 2020;382(25):2411-8.

26. Mehra MR, Desai SS, Ruschitzka F, Patel AN. RETRACTED: Hydroxychloroquine or chloroquine with or without a macrolide for treatment of COVID-19: a multinational registry analysis. Lancet. 2020 May 22:S01406736(20)31180-6

27. WHO Solidarity Trial Consortium et al. Repurposed Antiviral Drugs for Covid-19 - Interim WHO Solidarity Trial Results. N Engl J Med. 2021;384:497-511.

28. RECOVERY Collaborative Group, Horby P, Mafham M, Linsell L, Bell JL, Staplin N, Emberson JR, et al. Effect of hydroxychloroquine in hospitalized patients with Covid-19. N Engl J Med. 2020;383(21): 2030-40. 